\title{
Optimization of Erbium-Doped Actively Q-switched Fiber Laser Implemented in Symmetric Configuration
}

\author{
S.A. Kolpakov, S. Sergeyev, C. Mou, N. T. Gordon, and K. Zhou
}

\begin{abstract}
We report the results of an experimental study aimed at improving the performance of actively Q-switched fiber lasers. Unlike generic design schemes employing photonic crystal fibers, large modal diameter fibers or double-clad fibers, we demonstrate a high-power, actively $Q$-switched laser based on standard communication erbium doped fibers with peak irradiance beyond the state-of-the-art at $3.1 \mathrm{GW} / \mathrm{cm}^{2}$. The laser had $2.2 \mathrm{~kW}$ peak power, $15 \mathrm{~ns}$ pulse duration, $36.8 \mu \mathrm{J}$ pulse energy. We have also investigated the dynamics of pulse generation and have successfully suppressed pulse instabilities caused by backscattered laser emission reaching the pump laser diodes.
\end{abstract}

Index Terms-Fiber laser, optimization, giant pulse, active Q-switching, erbium doped fiber.

\section{INTRODUCTION}

Q UALITY-factor-switching fiber lasers (QS-FL) are devices that can generate extremely powerful light pulses [1]. While the QS-laser is pumped, energy, which is absorbed by the active laser element is stored. When the quality factor of the cavity (Q-factor) is switched, this stored energy can be discharged in one single pulse. The shape of the pulse will normally be either Gaussian or close to the Gaussian; however sometimes the generated pulse may be split into several subpulses (Q-switch self-mode-locking effect) [2] and this greatly reduces the pulse peak power.

During the three last decades the development of pulsed laser sources has made notable progress. Nevertheless, the main trend in the development of high-power pulsed laser sources has consisted in improving the laser material and pump system rather than in investigating the processes that take place inside of the laser resonator. As a result of these efforts photonic-crystal fibers of very complex design [3], large modal diameter fibers [4], [5] and double-clad fibers [6] have been demonstrated as laser components suitable for generating more powerful laser pulses. Some excellent results have been obtained using these laser components [7], [8], but still the availability of a such components is very poor. We have listed the characteristics of some relevant implementations of Qswitched erbium doped fibre lasers (QS-EDFLs) in Table I, for comparison with this work. In the table, the peak irradiance

This work was supported by West Midlands European Regional Development Fund (ERDF) project.

S. A. Kolpakov S. Sergeyev, C. Mou, N. T. Gordon, and K. Zhou are with the School of Engineering and Applied Science. Aston Triangle Birmingham, B4 7ET, (e-mail:stanislav.kolpakov@gmail.com, s.sergeyev@aston.ac.uk, c.mou1@aston.ac.uk,n.gordon@aston.ac.uk and k.zhou@aston.ac.uk).

Manuscript received November 30, 2013; revised, .
TABLE I: Features of $\mathrm{Er}^{3+}$ doped QS-FLs

\begin{tabular}{|c|r|c|c|c|}
\hline Peak Irrad., GW/cm & Pulse, ns & Ampl., kW & MA $^{(\mathbf{1})} \mathbf{~ n m}^{\mathbf{2}}$ & Ref. \\
\hline \hline 3.1 & 15 & 2.2 & 71 & $\mathrm{TW}^{(2)}$ \\
\hline 2 & 11 & 4.2 & 208 & {$[5]^{(3)}$} \\
\hline 1.1 & 100 & 3.5 & 314 & {$[10]^{(4)}$} \\
\hline 0.16 & 58 & 0.032 & 20 & {$[11]^{(5)}$} \\
\hline 0.07 & 12 & 0.05 & 71 & {$[12]^{(5)}$} \\
\hline
\end{tabular}

${ }^{(1)}$ Modal area

${ }^{(2)}$ This work

${ }^{(3)}$ Special Large Modal Area fibre

${ }^{(4)}$ Passive QS-EDFL

${ }^{(5)}$ Typical actively QS-EDFL based on communication fibres

of the different pulsed lasers are compared. In case of a laser that generates only a Gaussian-like mode, the average peak irradiance can be estimated as the ratio between the peak power and the modal area (MA).

The previous designs are still limited by the dependence of the pulsed laser performance on the level of the fiber doping and the cavity length. Previously we have shown that a proper understanding of the processes that take place inside the laser resonator allows the design of Q-switched laser where the pulse length does not depend on the resonator length [9]. In this paper we propose the concept of a laser which allows the generation of extremely bright pulses. We have achieved a pulse energy of $36.8 \mu \mathrm{J}$ and a peak irradiance of $3.1 \mathrm{GW} / \mathrm{cm}^{2}$, generated directly from the oscillator. Our results demonstrate that a theoretical approach based on the distributed theory of actively QS-EDFL allows the construction of lasers with the sufficient power to reach the micromachining threshold for metals, even using standard $\mathrm{Er}^{3+}$ doped telecommunication fibres that are usually considered inefficacious for such applications. This opens the way to avoid the dependence of the QS-FL performance on the active fiber length and doping level. Furthermore, it can eliminate the need for expensive chillers in powerful pulsed lases, as QS-FLs based on communication fiber do not need cooling.

In our earlier publication [13] we demonstrated that the ability of a laser to generate smooth pulses is linearly dependent on the ratio between the switching time of the acoustooptic modulator (AOM) and the time that the light takes for a round trip through the laser resonator. For Q-switched lasers in the classic implementation, the threshold ratio for smooth 
pulses is close to four [13]. This number means that for the laser to generate a smooth pulse, light needs to propagate around the resonator at least four times. The same ratio for a device implemented in the symmetric configuration is close to one [13]. This means that we can build a Q-switched laser with an approximately four times longer cavity and still obtain smooth pulses. The longer active cavity means either a longer active fiber or more active ions distributed along the cavity. That, in turn, means a larger energy-storing capability in the laser and consequently a more energetic pulse.

In this paper we have extended the study of Q-switched laser systems implemented in the symmetric configuration [13]. The optimization of the laser as a function of the length of the active fiber has been performed experimentally. It was found that the performance curve has a maximum with an active fiber length that corresponds to approximately $24 \mathrm{~dB}$ of amplification. This result is close to the accepted optimum active fiber length for pulse propagation in fiber amplifiers [14]. Exited state absorption (ESA), constitutes a very important source (more than $35 \%$ of energy) of losses in erbium doped fiber Q-switched lasers (QS-EDFL) [15]. The cavity losses due to modal diameter mismatch in fiber splices and those intrinsic to $\mathrm{AOM}$ were identified as the other relevant effects which limit the maximum pulse energy. The fiber mismatch losses can be minimized by the selection of an appropriate fiber but unfortunately the intrinsic AOM losses cannot have be reduced at least in this set of experiments.

Previously it was theoretically predicted [15] that maximum energy density that can be obtained from a QS-FL based on erbium doped fibers (QS-EDFL) could be increased approximately seven times by using the symmetrical configuration with a pump wavelength of $1480 \mathrm{~nm}$ and minimizing the losses on splices. In this paper we have experimentally demonstrated a pulse energy scaling of approximately six times which is close to the previous theoretical prediction. In addition, we have studied the pulse instabilities in the proposed laser scheme. Such unstable behavior typically appears when the amplification of the both laser arms is increased above a value of 17-18 dB. These instabilities were recognized as one of the main factors limiting the maximum achievable pulse energy and peak power. We have found experimentally, in our case, that these instabilities arise because of extremely powerful backscattered pulses that are able pass through both the wavelength-division multiplexer (WDM) and the isolator, and can disrupt the normal pump-diode operation. After the pump diode was properly protected from this backscattered radiation, these pulse instabilities were no longer observed.

We have demonstrated the principles of enhancing the power of fiber lasers using readily-available, low-cost, standard erbium doped fibers and have avoided the need for expensive special fibers. However, our aim in this paper was not to achieve the maximum peak power but to demonstrate experimentally that it is possible to find conditions where the discharge of the cavity will occur during the shortest possible time of 1.75 round-trips in our case. This minimization of the discharge time leads to the minimization of losses, greatly improving the QS-FL performance. Work is in hand to develop further. Finally, we note that the principles discussed in this article are expected to be valid for other types of fiber, solid state or another active laser material.

\section{EXPERIMENTAL SETUP}

In order to optimize the laser performance, a set of lasers with increasing active fiber (AF) lengths were built. The passive elements in the laser, such as FBGs tails and modulator passive fiber ends, were minimized and the lengths of these elements were kept constant in each experiment. Two versions of the laser design were compared; with and without protecting the pump diode against backscattered pulses. This allowed us to investigate the effect of backscattered pulses on the pulse stability. The QS-EDFL design without the diode protection is illustrated in Fig. 1. In this figure, EDF labels the active erbium doped fiber (which was Liekki80-8/125 fiber with a modal field diameter of $9.5 \mu \mathrm{m})$; and AOM refers to the acousto-optic modulator. This was a Gooch\&Housego modulator (model SFO2995-T-M080-0.4C2J-3-F2S) which was optimized for a central wavelength of $1532 \mathrm{~nm}$, had a $35 \mathrm{~ns}$ rise/fall time and an insertion loss of $2.9 \mathrm{~dB}$. This modulator was driven by an analogue modulator driver (Gooch\&Housego, model CHP080$3 A C-D 50,80 \mathrm{MHz}$ ) (not shown in Fig. 1) and controlled using a Stanford Research DG535/2 signal generator. This generator is able to generate rectangular pulses with tunable width and variable frequency.

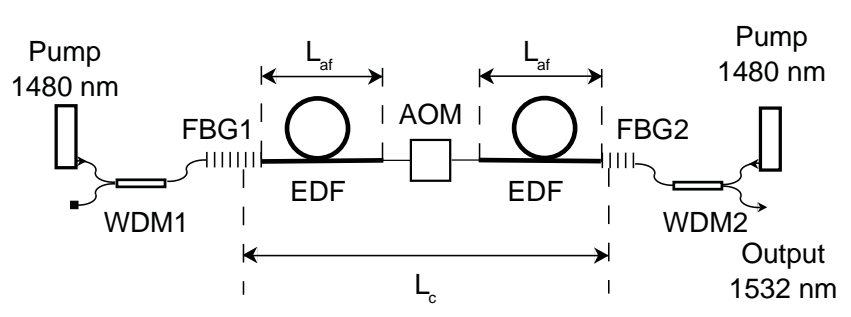

Fig. 1: The symmetric configuration of the QS-EDFL without diode protection.

The two pump diodes were supplied by Furukawa Electric. These were FOL1405RTD-317 series with a peak wavelength of $1480 \mathrm{~nm}$ and included an internal isolator to protect the diode against backscattered radiation. The diodes were driven with an Elliot Scientific LDR1500E driver. The fiber Bragg gratings (FBG1 and FBG2), which had a $1 \mathrm{~nm}$ bandwidth and reflectivities of $99 \%$ and of $7 \%$ respectively, were used as a cavity couplers. The reflectivity of the output mirror (FBG2) was selected following a theoretical optimization using the techniques discussed in references [9], [16]. It should be noted however that the reflectivity of the output coupler of the laser has not been optimized experimentally.

The output grating FBG2 was tuned in order to have the same central wavelength as FBG1 by stretching it using a Translation Stage with a travel range of $20 \mathrm{~mm}$. The laser output was measured with an ultra-fast InGaAs photodetector UPD-15-IR2-FC which had a rise time of $<15 \mathrm{ps,}$ a bandwidth $>25 \mathrm{GHz}$ and operated over the spectral range from $800 \mathrm{~nm}$ to $1700 \mathrm{~nm}$. The laser pulse was attenuated by 
figure) in front of the detector to prevent it being damaged by the pulse. The signal from the detector was recorded on an Agilent MSO9254A Infiniium MSO-2.5 GHz oscilloscope. The figure also shows two wavelength-division multiplexers labeled WDM1 and WDM2 which separated the pump and signal beams.

Fig. 2 shows the improved laser design with extra protection for the pump diodes. This also includes a module to study the dynamics of pulse formation.

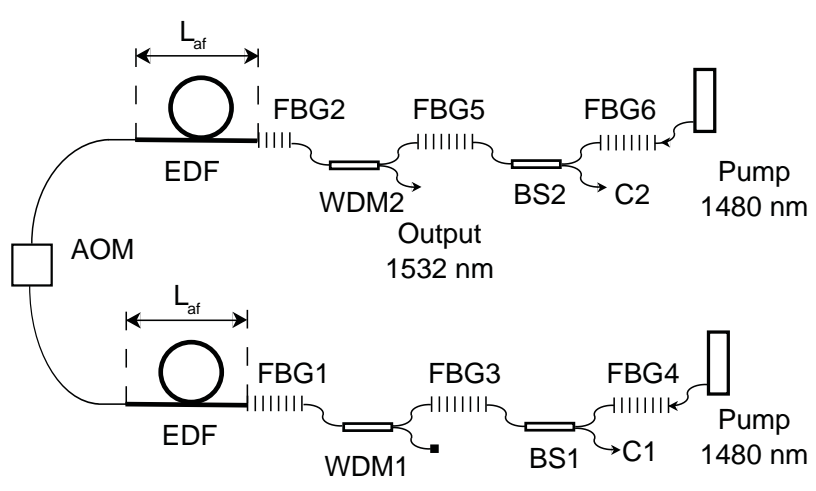

Fig. 2: QS-EDFL in the symmetric configuration with diode protection and the scheme to measure the pulse delay.

The purpose of the two measurement modules that are depicted in Fig. 2 was to allow measurement of delay between the AOM receiving an "on" trigger signal from the generator and the pulse being detected. The role of FBG3 and FBG5 was to attenuate the pulse to prevent saturation of the detector, while allowing the ASE to propagate through it. In this way we were able to record the wide pulse of ASE that appears when the AOM is switched "on" and the main laser pulse simultaneously as shown in Fig. 5 and Fig. 6. The pulse amplitude emitted from the end of the laser with the highly reflective FBG (FBG1) is $\sim 30$ times smaller than the pulse emitted from the laser output coupler with FBG2. Thus greater attenuation is needed to protect the detector at the FBG2 end. Because of this, the attenuation of the FBG5 grating was chosen to be $27 \mathrm{~dB}$; and the attenuation of FBG3 was chosen to be $18 \mathrm{~dB}$. In Fig. 2 Symbols BS1 and BS2 denote broad band beam splitters 10 : 90 with a central wavelength of $1550 \mathrm{~nm}$. The first control port (C1) was used to monitor the delay between the AOM being switched and the output pulse reaching the detector. The second control output (C2) was used to check residual pulses propagated through the WDM2 and also to measure the output pulse delay relative to the moment when AOM switches between the "off" and "on" states. During the experiments we discovered that the combination of the isolator that was incorporated with the pump diode and the WDM were insufficient to attenuate the backscattered pulse. Because of this, two protection FBG (FBG4 and FBG6) were incorporated into the design to protect the pump diodes properly from backscattered pulses. Both FBGs had $99 \%$ reflectivity at $1532 \mathrm{~nm}$ and a bandwidth of $50 \mathrm{~nm}$. Experimentally it was found that the output pulse
FBGs 3 - 6 and BSs 1, 2. This can be explained by pump absorption in the splices between these new elements.

\section{EXPERIMENTAL RESULTS}

1) Study of instabilities of the pulses: The lasers that were built without the pump diodes protection FBGs (as shown in Fig. 1) were found to exhibit unstable behavior at certain pulse repetition frequencies. In our experiments, this unwanted chaotic behavior was found to be the major factor limiting the pulse repetition frequency. Typical unstable behavior for the laser that was built, with an active fiber length $\mathrm{L}_{\mathrm{af}}=50 \mathrm{~cm}$ and a total cavity length $\mathrm{L}_{\mathrm{c}}=174 \mathrm{~cm}$, is illustrated in Fig. 3. This shows how the peak power and average power output of the laser vary with repetition rate. When the pulse repetition rate was increased towards $7.5 \mathrm{kHz}$, the peak was suddenly split in four (see insert in Fig. 3). At this frequency, Fig. 3 shows a discontinuity in both the average power and the peak power of the laser. Further experiments have shown that the frequency of this transition to chaotic behavior strongly depends on the active fiber length, the pump power and the amplitude of the backscattered pulses. Monitoring of these backscattered pulses shows that their amplitude can achieve hundreds of Watts while the peak power of laser-generated pulses is more than $2 \mathrm{~kW}$. When the FBG4 and FBG6 were included in the laser scheme (Fig. 2) it became possible to manufacture higher performance lasers with longer active fibers.

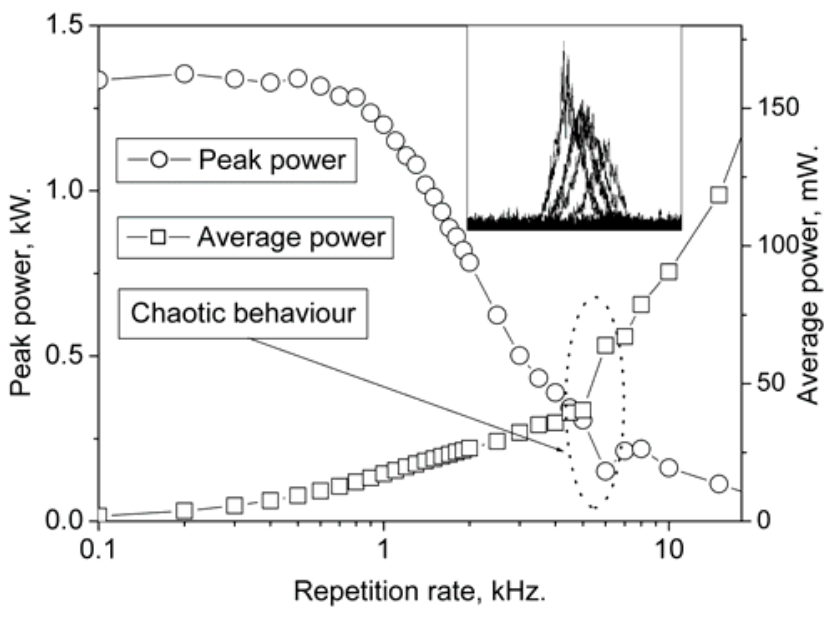

Fig. 3: Evidence of chaotic regime in QS-EDFL with $2 \times 50$ $\mathrm{cm}$ active segments. Chaotic behavior appears at a frequency of $7.5 \mathrm{kHz}$ where the pulse splits into four (see insert). This characteristic curve was measured in the laser without diode protection. The estimated pulse peak power was measured to be almost $500 \mathrm{~W}$ (first peak).

Moreover, monitoring the backscattered pulses through the ports $\mathrm{C} 1$ and $\mathrm{C} 2$ showed that the pulse amplitude reaching the diode had been reduced to less than 1 Watt. The behavior of both pump diodes become stable and the chaotic behavior disappeared.

The performance curves of the laser with the protection 


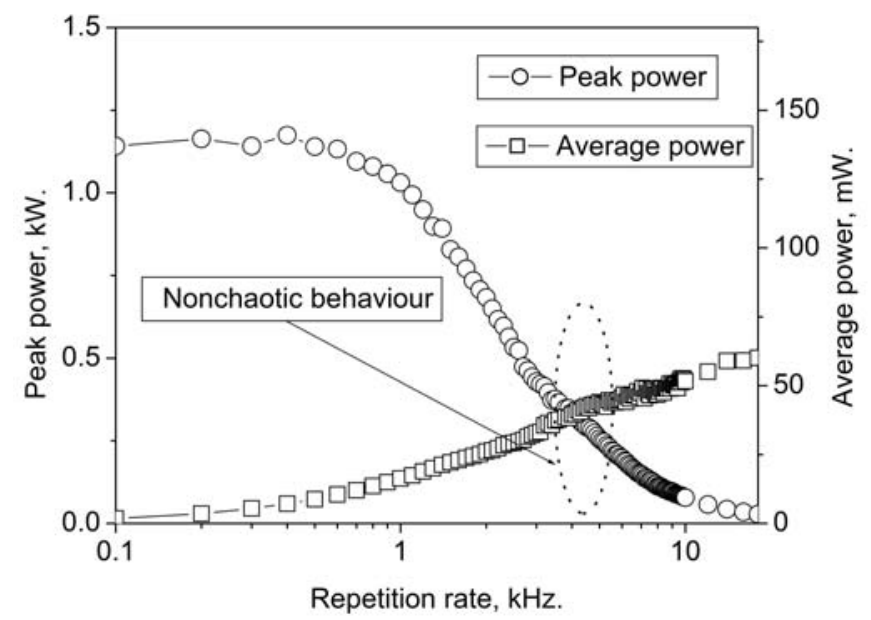

Fig. 4: Characteristic curves of the same laser as in Fig. 3 but with the pump diodes protected from backscattered laser pulses. The chaotic behavior shown in Fig. 3 has been eliminated.

and Fig. 4 shows that the chaotic laser behavior has been successfully eliminated. We therefore conclude that these experiments have shown that the origin of chaotic QS-laser behavior at certain frequencies in this symmetric QS-FL scheme is due to disruption of the normal operation of the pump diodes by residual pulses rather than due to the intrinsic properties of the active fiber material or laser resonator.

2) Measurement of the delay of a pulse formation: Port $\mathrm{C} 1$ illustrated in Fig. 2 was used to measure the pulse delay after switching the AOM. The spectral widths of FBG1 and FBG3 were $1 \mathrm{~nm}$ and $20 \mathrm{~nm}$ respectively and this allowed some of ASE to pass through and be detected by the detector. A small fraction of the pulse energy was able pass through both Bragg gratings, WDM1 and BS1 and be detected on port C1.

Fig. 5 illustrates the signal that was recorded from port $\mathrm{C} 1$ during operation of a laser with a cavity length of $155 \mathrm{~cm}$ including two segments of active fiber, each with a length of $30 \mathrm{~cm}$.

In Fig. 5, label "1" shows the detected ASE radiation. The AOM receives the driving signal at the time labeled "2" and becomes fully opened 35 ns later ("3"). The label "4" indicates the moment when AOM is closed again. Finally, markers "5" and " 6 " label the main pulse and small replicas that appear because of the Fabry-Perot resonator that is formed between FBG1, FBG3 and FBG4. These replicas are decay very quickly because the quality factor of this resonator is quite low. Once the modulator is completely open (3) and before the pulse begins (5), the ASE intensity decreases rapidly (7). This fading is the result of energy transfer from ASE to signal. During this process, the signal intensity is growing very quickly due to absorbtion of energy that was stored in the active core and this reduces the gain for ASE.

In Fig. 6 pulses recorded from port $\mathrm{C} 1$ are plotted on a

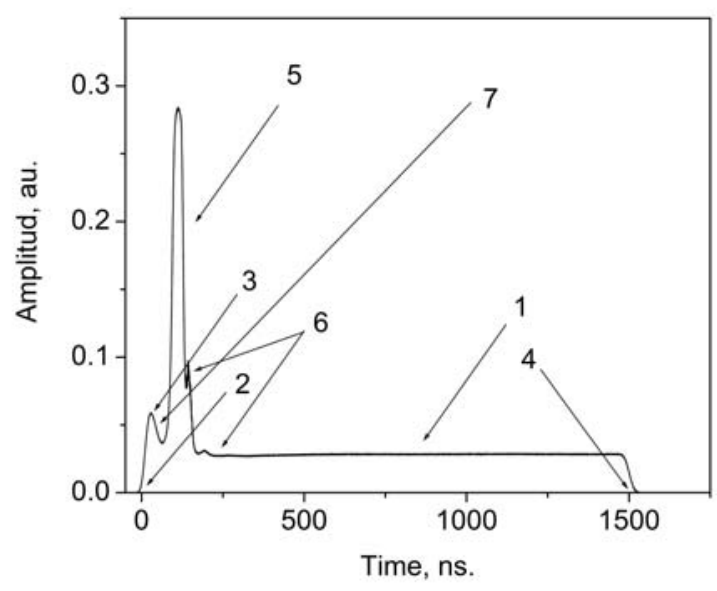

Fig. 5: Illustration showing the main features of the pulses.

The results are labeled "1" for the laser with an active fiber length of $\mathrm{L}_{\mathrm{af}}=70 \mathrm{~cm}$ and total cavity length of $\mathrm{L}_{\mathrm{c}}=215 \mathrm{~cm}$, "2" and "3" for the lasers with $\mathrm{L}_{\mathrm{af}}=80 \mathrm{~cm}\left(\mathrm{~L}_{\mathrm{c}}=235 \mathrm{~cm}\right)$ and $\mathrm{L}_{\mathrm{af}}=100 \mathrm{~cm}(\mathrm{Lc}=275 \mathrm{~cm})$ respectively. Symbols "A" and "B" show replicas of the main pulse (see position (6) in Fig. 5). At time zero, the AOM is completely closed and will have a very low transmittance of $-40 \mathrm{~dB}$. The vertical dotted line labeled "35 ns" shows the time when AOM is completely open and its transmittance is increased to $-2.9 \mathrm{~dB}$. In the first case, the length of the active fiber is significantly shorter than the optimal length and the round-trip period is $21.5 \mathrm{~ns}$. The maximum of the pulse $(71 \mathrm{~ns})$ is delayed 3.3 round-trips from the moment when the modulator receives the driving pulse.

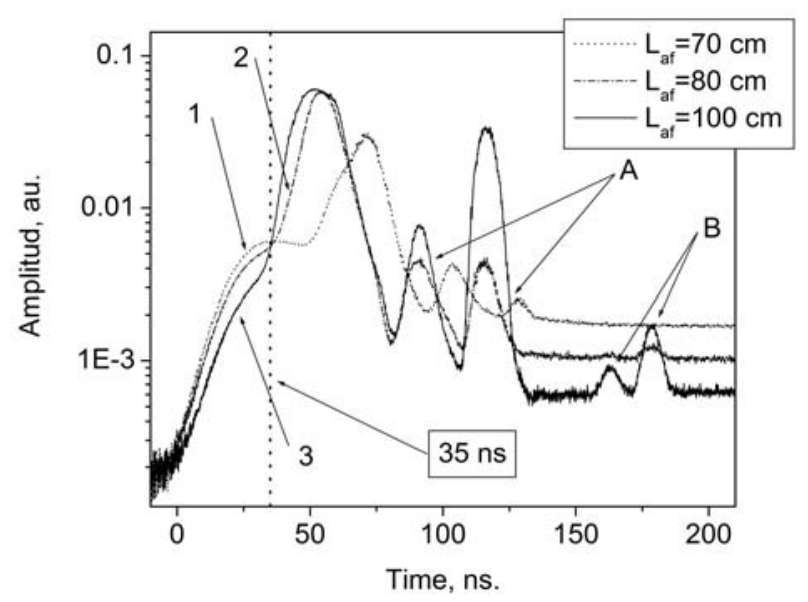

Fig. 6: Different pulse formation behavior for three lasers with: (1) $70 \mathrm{~cm}$; (2) $80 \mathrm{~cm}$; and (3) $100 \mathrm{~cm}$ sections of active fiber.

In the second case where the active fiber length was $80 \mathrm{~cm}$ ("2" in Fig. 6), the pulse maximum is at $55 \mathrm{~ns}$ and is delayed by 2.3 round-trips (the round-trip period in this case is $23.5 \mathrm{~ns}$ ) after triggering the modulator. Finally, in the third case ("3" 
pulse maximum is delayed by $\sim 1.88$ round-trips. The delay is not an integer number of round-trips because during the first $\sim 6$ ns the AOM transmittance is not sufficient to produce ASE perturbation in the laser cavity because the ASE power transmitted from opposite arm is very low in comparison with ASE power generated by active fiber. As a result the pulse edge in a Q-switched laser is always delayed by this additional offset. That means than after subtraction of the first $\sim 6 \mathrm{~ns}$, the real delay of the pulse maximum is approximately 1.66 round-trip periods. And the width of the pulse base is $\sim 1.65$ round-trips

The idealized and linear model of a QS-FL in a symmetric configuration predicts that the pulse starts to form when the first photon of the perturbation is formed when AOM begins switching "on" reaches the output mirror. If we suppose that the AOM switches instantly, that time will be one quarter of the round-trip period. The formation of the pulse will be completed when the last photon of the counter-propagated perturbation completes one full round-trip and reaches the output mirror. In this case the time will be 1.75 round-trip periods. Accordingly, the minimal delay of the pulse should be 0.25 round-trips (around $14 \mathrm{~ns}$ in the experiment) and maximum pulse width in the base of the pulse needs to be 1.5 round-trips. (This was $\sim 1.65$ round-trips in the experiment giving a pulse width of $\sim 50 \mathrm{~ns}$ rather than $\sim 40 \mathrm{~ns}$ in ideal case.) In the real situation the optimum pulse widths will be slightly different because of finite time of AOM switching and the effect of passive losses in the cavity. The role played by ion-ion up-conversion also may be important and needs to be investigated theoretically [17], [18].

3) Optimization of Laser Scheme: Optimization of the laser output was investigated using the improved symmetric design discussed earlier which eliminates chaotic behavior. The lengths of both active fibers were changed simultaneously to maintain a symmetric design. At the same time, the lengths of the passive elements such as FBG1, FBG2 and AOM passive fiber tails (SMF-28) were maintained constant and kept as short as possible. The laser pulse frequency was fixed at $100 \mathrm{~Hz}$. The dependence of the peak power, pulse energy and pulse width on the length of the active fiber $L_{a f}$ are illustrated in Figs. 7-9 respectively. In all these figures, the "diamond" symbol shows the results that were obtained for the lasers without FBG4 and FBG6 (Fig. 1); the "star" symbol shows the results from the laser with diode protection and using a pump power of $200 \mathrm{~mW}$ from each pump diode. Finally, the "circles" show the results from the laser with diode protection and using a pump power of $260 \mathrm{~mW}$ from each diode. The labels "1" and "2" indicate the laser schemes before and after adding the protection to the pump diodes. The value of $260 \mathrm{~mW}$ was the maximum pump power that the pump diodes were able to deliver to the laser cavity. The pump power was slightly reduced by losses in the beam splitter (BS) and splices between the diode-protecting FBGs and the maximum pump power was limited by overheating of diodes.

The dependence of the peak power on the active fiber length is illustrated in Fig. 7. The experiments show a maximum in $\mathrm{cm}$ and achieve maximum peak powers of $1.35 \mathrm{~kW}$ and 2.2 $\mathrm{kW}$ for pump powers of $200 \mathrm{~mW}$ and of $260 \mathrm{~mW}$ respectively (Fig. 7).

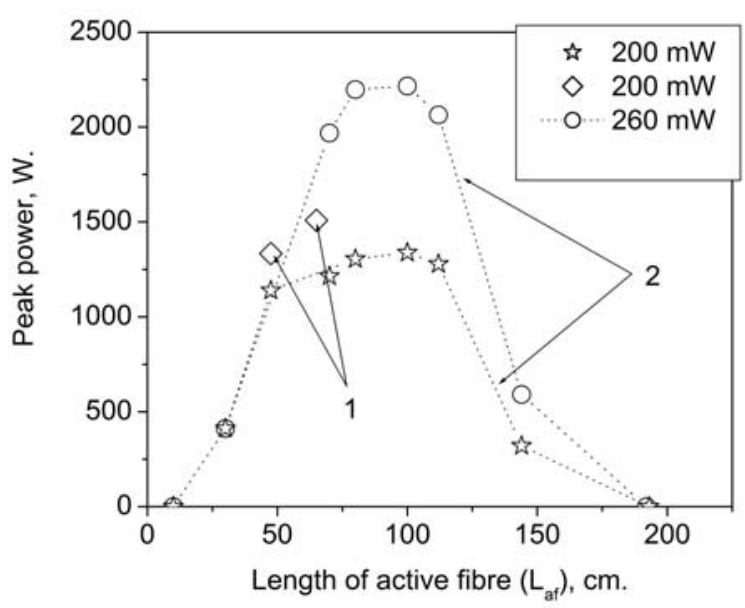

Fig. 7: Generated peak power as a function of the active fiber length.

Comparison of the performance of the designs without diode protection (diamonds) with the performance of the designs with diode protection and measurement module and the same pump conditions (stars) show a small reduction in the output power and pulse energy when the additional FBG3 - FBG6 and BS1 and BS2 were attached. The reason for this is the absorption of some of the pump energy in the splices between added elements. This has been verified experimentally; the losses in the protecting scheme were compensated by increasing the power delivered by the two pump diodes. When the optical power delivered to the cavity was matched for the two cases, the peak power, peak energy and peak shape were the same.

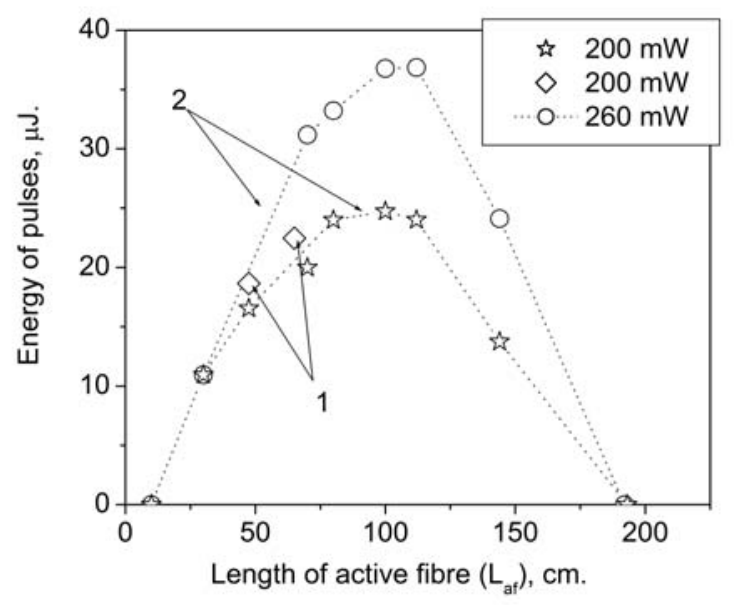

Fig. 8: Energy of pulses as a function of the active fiber length.

Furthermore, when the pump power was increased, the 
These results are thought to be due to by ion-ion interaction in highly doped Liekki Er80 fiber [17].

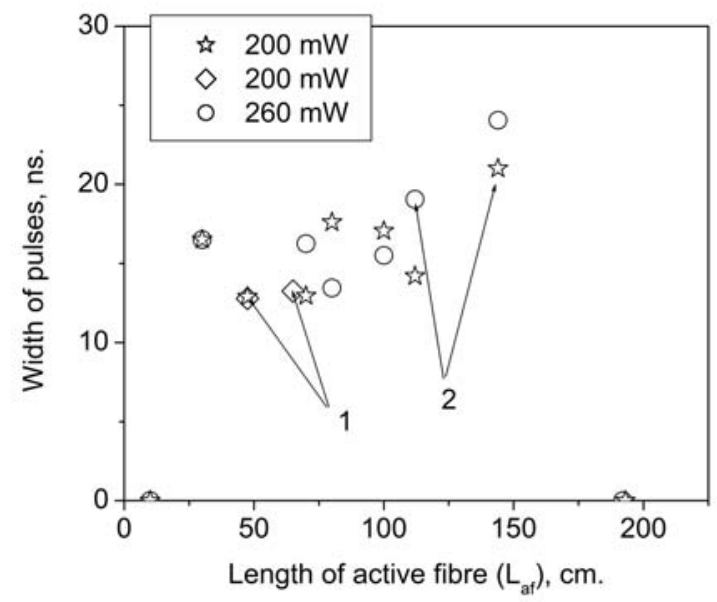

Fig. 9: Width of pulses as a function of the active fiber length.

Finally, comparison of the width of the pulses generated in the different regimes (Fig. 9) shows that the pulse width is relatively unaffected by the active fiber length (symbol "1"), except for the situation in very long active fiber where it begins to absorb energy (symbol "2"). This absorption is equivalent to additional passive losses in the cavity which generate wider pulses [16].

4) Characterization of pulses: Pulses generated by the setup depicted in Fig. 2 with an $80 \mathrm{~cm}$ active fiber length and pump power of $260 \mathrm{~mW}$ are shown in Fig. 10. This length of active fiber is close to the optimal condition (see Fig. 7 Fig. 9). The pulses produced at a repetition rate of $100 \mathrm{~Hz}$ have an amplitude of $2.2 \mathrm{~kW}$ and a pulse width of $13.5 \mathrm{~ns}$, measured at the half-of-the-peak amplitude. The pulse energy was estimated to be of $30 \mu \mathrm{J}$.

For higher frequencies the peak becomes lower and wider (see Fig. 10-Fig. 11). This is typical behavior for a QS-laser. The explanation of this behavior is that a certain amount of time is necessary to completely recharge the fiber between two pulses. When the pump power is insufficient to recharge the fiber completely, the energy delivered in each laser pulse diminishes. Moreover, the partially recharged fiber acts as the source of additional losses in the laser cavity. This is because the fiber will begin to absorb signal instead of amplifying it during the final stage of pulse formation [16].

The pulses, generated in the same conditions by the laser with an active fiber length $\mathrm{L}_{\mathrm{af}}=100 \mathrm{~cm}$ in each cavity, are illustrated in Fig. 11. The pulses are wider and more energetic than in the previously discussed case. For a repetition rate of $100 \mathrm{~Hz}$, pulse energy was measured to be $36.8 \mu \mathrm{J}$. This was the most energetic pulse obtained during all the sets of experiments. The peak profiles for both this and the previous figure were averaged over 16 pulses.

5) Jitter and noise: It was not possible to analyze the pulse

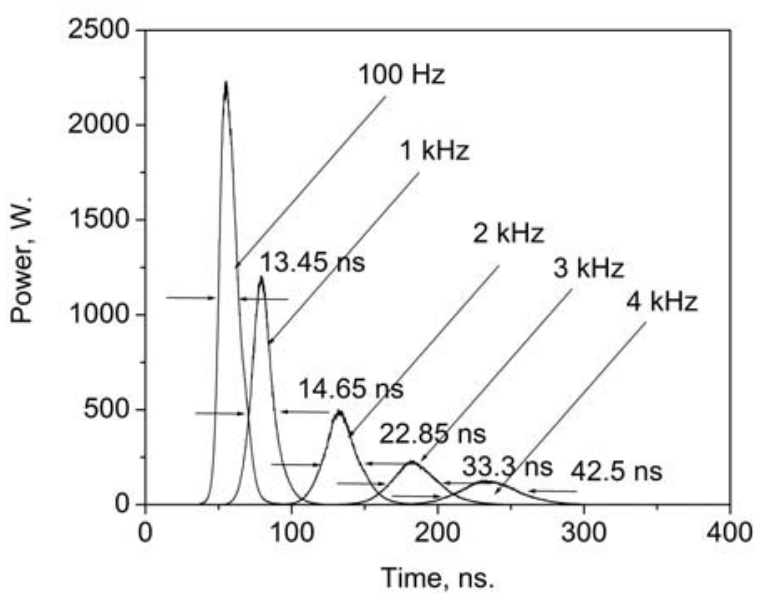

Fig. 10: Pulses generated using an active fiber segment length $\mathrm{L}_{\mathrm{af}}=80 \mathrm{~cm}$ and a pump power of $260 \mathrm{~mW}$ (from each pump diode).

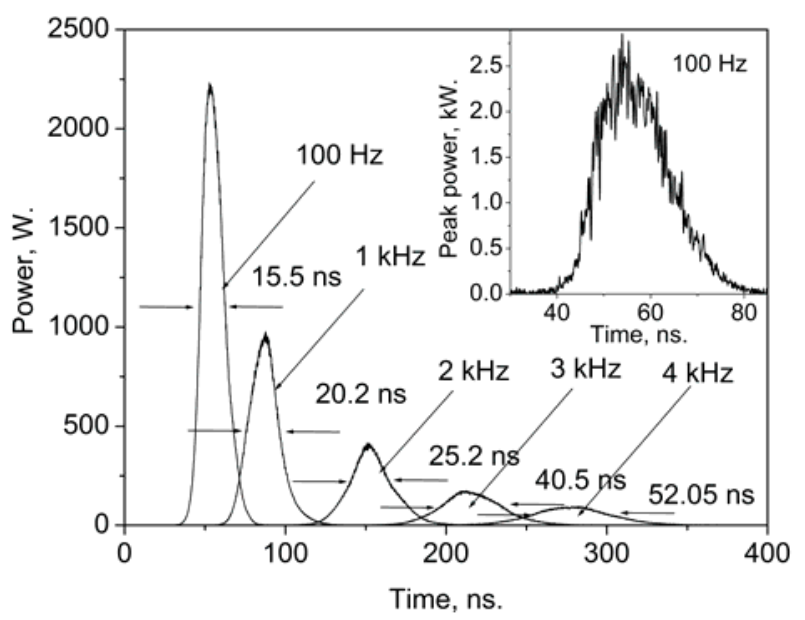

Fig. 11: Pulses generated using an active fiber segment length $\mathrm{L}_{\mathrm{af}}=100 \mathrm{~cm}$ and a pump power of $260 \mathrm{~mW}$ (from each pump diode). Insert illustrates the pulse without averaging obtained for $100 \mathrm{~Hz}$ of repetition rate.

of our oscilloscope. However the pulse train could be analyzed at a repetition rate of $800 \mathrm{~Hz}$ and this is shown in Fig. 12. At this repetition rate, the peaks were still similar to the peaks obtained at $100 \mathrm{~Hz}$ (Fig. 4). Due to the high levels of ASE noise inherent to pumping at a wavelength of $1480 \mathrm{~nm}$, the pulse train shows a variation in the peak amplitude from pulse to pulse, and this is also evident in Fig. 13.

The infinite persistent figure which is illustrated in Fig. 13 shows that the pulse is quite noisy and has a temporal jitter that is bigger than the jitter of the signal generator. Comparison of this figure with the Fig. 6 of [13] shows that at a pump wavelength of $980 \mathrm{~nm}$ the jitter is $\sim 20 \%$ less than at the wavelength of $1480 \mathrm{~nm}$ and the amplitude of the pulses at the shorter pump wavelength are more uniform in height. 


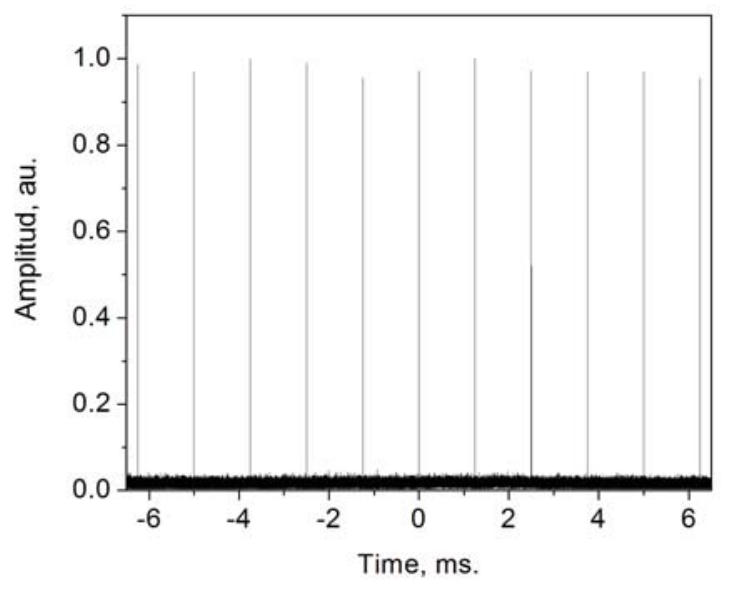

Fig. 12: Pulse train.

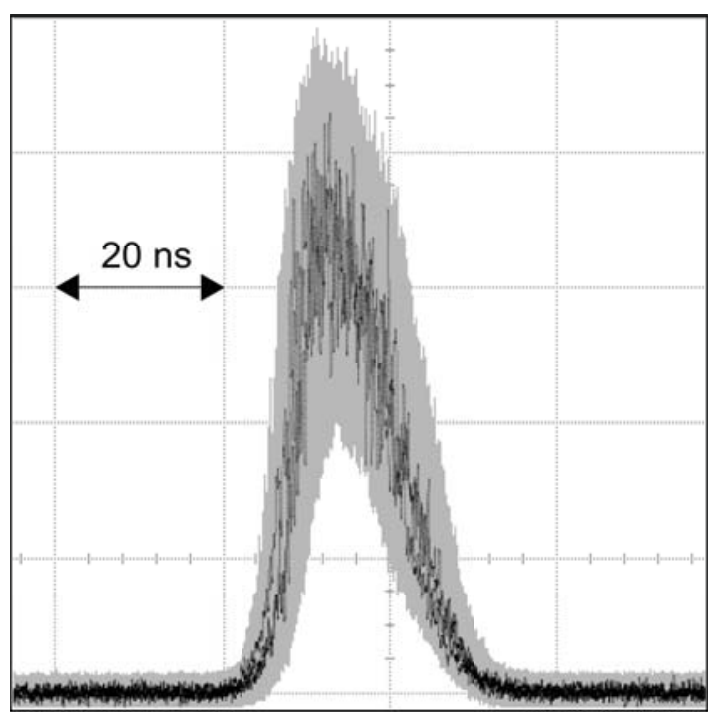

Fig. 13: Infinite persistent figure showing the jitter and amplitude variation between pulses.

generate significantly more noise when pumped at $1480 \mathrm{~nm}$.

6) Spectral Characteristics: Spectra obtained from a QS-EDFL in the symmetric configuration are shown in Fig. 14 for three different repetition frequencies $(1 \mathrm{kHz}, 4 \mathrm{kHz}$ and $10 \mathrm{kHz})$. The spectral width $(1 \mathrm{~nm})$ is determined by the spectral width of the laser couplers (FBGs). The solid line shows the spectrum for $1 \mathrm{kHz}$ repetition frequency. At this frequency, the amplified spontaneous emission spectrum is still visible for wavelengths away from the peak, because the active fibers will acquire considerable charge during the relatively long temporal interval between pulses. At this repetition frequency, the generated pulses still have considerable amplitude ( Fig. 10 ). The dashed line shows the spectrum at $4 \mathrm{kHz}$. In this situation the spontaneous emission spectrum is largely eliminated however the pulse amplitude and energy is also significantly diminished ( compare with Fig. 4 and Fig. 10 ).

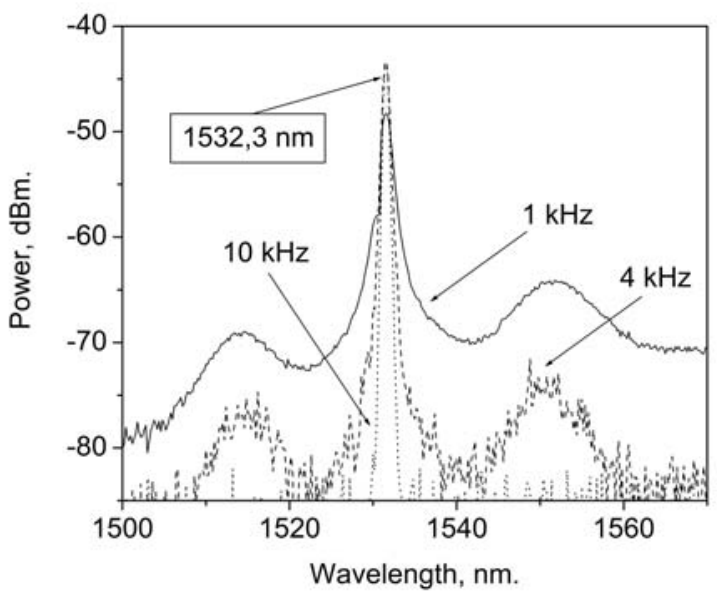

Fig. 14: Spectra recorded for $1 \mathrm{kHz}$ (solid line), $4 \mathrm{kHz}$ (dashed line) and $10 \mathrm{kHz}$ (dotted line). The central wavelength is $1532.5 \mathrm{~nm}$.

repetition frequency. As can be seen in Fig. 4, pulses that are generated at this frequency have a very small energy and low amplitude. In this situation, the supplied pump power is not sufficient to recharge the fiber during the interval between two adjacent pulses. In this scenario the level of ASE is very low because the population of the second level is very close to $50 \%$ and practically all the stored energy is transformed to radiation at the $1532.5 \mathrm{~nm}$ main wavelength. Spectral studies of RF frequency also have shown a complete absence of the radio-frequency components in the laser spectra.

\section{CONCLUSION}

We have demonstrated the principles of enhancing the power of actively QS-FL using readily-available, low-cost, standard erbium doped fibers. Experiments found that the optimal active fiber length was $100 \mathrm{~cm}$ and maximum peak powers of 1.35 $\mathrm{kW}$ and $2.2 \mathrm{~kW}$ were achieved for supplied pump powers of $200 \mathrm{~mW}$ and $260 \mathrm{~mW}$ respectively. Moreover, we have studied the dynamics of pulse generation and demonstrate experimentally that in the presented QS-EDFL the cavity is discharged during the time that is close to minimal $(\sim 1.85$ round-trips in the experiment versus 1.5 round-trips in the ideal case). As was expected, the minimization of the discharge time notably improved the laser performance. This suggested design for a high power actively QS-FL is a cost-effective technology that can find applications in different fields of science and engineering including laser material ablation [19], laser assisted micro-machining [20], supercontinuum generation [21], LIDAR [22] and bio-medicine [23]. We expect that the ideas presented in this article can also be applied to actively QS lasers that are based on other types of active fibers as well as to solid state and gas lasers.

\section{ACKNOWLEDGMENT}

The authors would like to thank Professor Sergei K. Turitsyn 


\section{REFERENCES}

[1] J. W. Dawson, M. J. Messerly, H. H. Phan, J. K. Crane, R. J. Beach, C. W. Siders, and C. Barty, "High-energy, short-pulse fiber injection lasers at lawrence livermore national laboratory," IEEE Journal of Selected Topics in Quantum Electronics, vol. 15, no. 1, pp. 207-219, 2009. [Online]. Available: http://dx.doi.org/10.1109/JQE.2011.2143695

[2] P. Myslinski, J. Chrostowski, J. Koningstein, and J. R. Simpson, "Selfmode locking in a q-switched erbium-doped fiber laser," Applied Optics, vol. 32, no. 3, pp. 286-290, 1993.

[3] P. Kadwani, N. Modsching, R. A. Sims, L. Leick, J. Broeng, L. Shah, and M. Richardson, "Q-switched thulium-doped photonic crystal fiber laser," Optics Letters, vol. 37, no. 10, pp. 1664-1666, 2012. [Online] Available: http://dx.doi.org/10.1364/OL.37.001664

[4] R. Naftali, B. Fischer, and J. R. Simpson, "Large core-area erbiumdoped fiber laser," Optics Communications, vol. 149, no. 4-6, pp. 317-320, 1998. [Online]. Available: http://dx.doi.org/10.1016/S00304018(98)00017-0

[5] G. P. Lees, D. Taverner, D. J. Richardson, L. Dong, and T. P. Newson, "Q-switched erbium doped fibre laser utilising a novel large mode area fibre," Electronics Letters, vol. 33, no. 5, pp. 393-394, 1997.

[6] A. S. Kurkov, Y. E. Sadovnikova, A. V. Marakulin, and E. M. Sholokhov, "All fiber er-tm q-switched laser," Laser Physics Letters, vol. 7, no. 11, pp. 795-797, 2010. [Online]. Available: http://onlinelibrary.wiley.com/doi/10.1002/lapl.201010066/pdf

[7] J. Alvarez-Chavez, H. Offerhaus, J. Nilsson, P. W. Turner, W. A Clarkson, and D. J. Richardson, "High-energy, high-power ytterbiumdoped q-switched fiber laser," Optics Letters, vol. 25, no. 1, pp. 37-39, 2000. [Online]. Available: http://dx.doi.org/10.1364/OL.25.000037

[8] S. D. Setzler, M. P. Francis, Y. E. Young, J. R. Konves, and E. P. Chicklis, "Resonantly pumped eyesafe erbium lasers," IEEE Journal of Selected Topics in Quantum Electronics, vol. 11, no. 3, pp. 645-647, 2005.

[9] S. A. Kolpakov, Y. O. Barmenkov, A. V. Kiryanov, A. D. GuzmnChvez, J. L. Cruz, and M. V. Andres, "Comparison of asymmetric and symmetric cavity configurations of erbium-doped fiber laser in active q-switched regime," JOSA B, vol. 29, no. 9, pp. 2453-2461, 2012. [Online]. Available: http://dx.doi.org/10.1364/JOSAB.29.002453

[10] A. Kurkov, Y. Sadovnikova, A. Marakulin, and E. Sholokhov, "All fiber er-tm q-switched laser," Laser Physics Letters, vol. 7, no. 11, pp. 795797, 2010. [Online]. Available: http://dx.doi.org/10.1002/lapl.201010066

[11] Y. M. Chang, J. Lee, Y. M. Jhon, and J. H. Lee, "Active q-switching in an erbium-doped fiber laser using an ultrafast silicon-based variable optical attenuator," Opt. Express, vol. 19, no. 27, pp. 26911-26916, Dec 2011. [Online]. Available: http://www.opticsexpress.org/abstract.cfm?URI=oe19-27-26911

[12] Z. Yu, M. Malmström, O. Tarasenko, W. Margulis, and F. Laurell, "Actively q-switched all-fiber laser with an electrically controlled microstructured fiber," Opt. Express, vol. 18, no. 11, pp. 11052-11057, May 2010. [Online]. Available: http://www.opticsexpress.org/abstract.cfm?URI=oe-18-11-11052

[13] S. A. Kolpakov, Y. O. Barmenkov, A. Kir'yanov, L. Escalante-Zarate, J. L. Cruz, and M. V. Andres, "Smooth pulse generation by a Q-Switched erbium doped fiber laser," IEEE Photonics Technology Letters, vol. 25, no. 5, pp. 480-483, 2013. [Online]. Available: http://dx.doi.org/10.1109/LPT.2013.2241048

[14] M. J. F. Digonnet, Ed., Rare-Earth-Doped Fiber Lasers and Amplifiers. Marcel Dekker, Inc., 2001

[15] Y. O. Barmenkov, S. A. Kolpakov, A. V. Kir'yanov, L. EscalanteZarate, J. L. Cruz, and M. V. Andres, "Influence of cavity loss upon performance of Q-Switched Erbium-Doped fiber laser," IEEE Photonics Technology Letters, vol. 25, no. 10, pp. 977-980, 2013. [Online]. Available: http://dx.doi.org/10.1109/LPT.2013.2257725

[16] S. A. Kolpakov, Y. O. Barmenkov, A. D. Guzman-Chavez, A. V. K J. L. Cruz, A. Diez, and M. V. Andres, "Distributed model for actively Q-Switched Erbium-Doped fiber lasers," IEEE Journal of Ouantum Electronics, vol. 47, no. 7, pp. 928-934, 2011. [Online]. Available: http://dx.doi.org/10.1109/JQE.2011.2143695

[17] S. Sergeyev, S. Popov, and A. Friberg, "Influence of the shortrange coordination order of erbium ions on excitation migration and upconversion in multicomponent glasses." Optics letters, vol. 30, no. 11, pp. 1258-1260, 2005. [Online]. Available: http://dx.doi.org/10.1364/OL.30.001258

[18] S. Sergeyev, "Model of high-concentration erbium-doped fibre amplifier: Effects of migration and upconversion processes." Electronics Letters, vol. 39, no. 6, pp. 511-512, 2003. 「Onlinel. Available:
[19] A. A. Oraevsky, L. B. D. Silva, A. M. Rubenchik, M. D. Feit M. E. Glinsky, M. D. Perry, B. M. Mammini, W. Small, and B. C. Stuart, "Plasma mediated ablation of biological tissues with nanosecond-to-femtosecond laser pulses: relative role of linear and nonlinear absorption." IEEE Journal of Selected Topics in Quantum Electronics, vol. 2, no. 4, pp. 801-809, 1996. [Online]. Available: http://dx.doi.org/10.1109/2944.577302

[20] W. O'Neill and L. Kun, "High-quality micromachining of silicon at $1064 \mathrm{~nm}$ using a high-brightness mopa-based 20-w yb fiber laser." IEEE Journal of Selected Topics in Quantum Electronics, vol. 15, no. 2, pp. 462-470, 2009. [Online]. Available: http://dx.doi.org/10.1109/JSTQE.2009.2012269

[21] A. S. Kurkov, E. M. Sholokhov, and Y. E. Sadovnikova, "Allfiber supercontinuum source in the range of $15502400 \mathrm{~nm}$ based on telecommunication multimode fiber." Laser Physics Letters, vol. 8, no. 8, p. 598600, 2011. [Online]. Available: http://dx.doi.org/10.1109/JSTQE.2004.835296(410) 1

[22] L. Fan-Yi and L. Jia-Ming, "Measurements of wind and turbulence profiles with scanning doppler lidar for wind energy application." IEEE Journal of Selected Topics in Quantum Electronics, vol. 10, no. 5, pp. 991-997, 2004. [Online]. Available: http://dx.doi.org/10.1109/JSTQE.2004.835296(410) 1

[23] N. J. Scott, R. A. Barton, A. L. Casperson, A. Tchapyjnikov, K. Levin, D. Tran, and N. M. Fried, "Mid-IR germanium oxide fibers for contact erbium laser tissue ablation in endoscopic surgery," IEEE Journal of Selected Topics in Quantum Electronics, vol. 13, no. 6, pp. 1709-1714, 2007. [Online]. Available: http://dx.doi.org/10.1109/JSTQE.2007.910557 